\title{
Myocardial Infarction as An Independent Predictor of Intramyocardial Hemorrhage in Acute Reperfused Myocardial Ischemic Rats
}

\section{Rui Xia ( $\nabla$ ruix2006@163.com )}

The First Affiliated Hospital of Chongqing Medical University

Tong Zhu

Tongji Hospital of Tongji Medical College of Huazhong University of Science and Technology

Yu Zhang

West China Hospital of Medicine: Sichuan University West China Hospital

Bo He

West China Hospital of Medicine: Sichuan University West China Hospital

Yushu Chen

West China Hospital of Medicine: Sichuan University West China Hospital

Lei Wang

West China Hospital of Medicine: Sichuan University West China Hospital

Yang Zhou

Chongqing Medical University

Jichun Liao

The First Affiliated Hospital of Chongqing Medical University

Jie Zheng

Washington University School of Medicine in Saint Louis: Washington University in St Louis School of Medicine

Yongmei Li

The First Affiliated Hospital of Chongqing Medical University

Fajin Lv

The First Affiliated Hospital of Chongqing Medical University

\section{Fabao Gao}

West China Hospital of Medicine: Sichuan University West China Hospital

\section{Research}

Keywords: intramyocardial hemorrhage, myocardial infarction, magnetic resonance imaging

Posted Date: October 28th, 2021

DOI: https://doi.org/10.21203/rs.3.rs-1008769/v1

License: () (1) This work is licensed under a Creative Commons Attribution 4.0 International License. Read Full License 


\section{Abstract}

Background: In the previous studies, hemorrhage occurred only with the largest infarctions and studies confirmed a moderate correlation between the extent of necrosis and hemorrhage, but the extent of infarction size of these studies was limited. This study was to find the correlations between intramyocardial hemorrhage (IMH), myocardial infarction (MI), and myocardial edema (ME) from days 2 to 7 in a 7.0T MR scanner.

Methods: The different degrees of myocardial ischemia were induced by occluding different sections of the proximal left anterior descending coronary artery (1-3 mm under the left auricle). T2*-mapping, T2-mapping and late gadolinium enhancement (LGE) sequences were performed on a 7.0T MR system at day 2 and 7. T2*- and T2-maps were calculated using a custom-made software. All areas were expressed as a percentage of the whole myocardial tissue of the left ventricle. The rats were divided into two groups based on the T2* results; $\mathrm{MI}$ with IMH was referred to as the +IMH group, while MI without IMH was referred to as the -IMH group.

Results: The final experimental sample consisted of 25 rats in the +IMH group and 10 rats in the $-I \mathrm{MH}$ group. For the +IMH group. On day 2, There was a significant positive correlation between IMH size and $\mathrm{MI}$ size $(r=0.677, \mathrm{P}<0.01)$, and a positive correlation between IMH size and ME size $(r=0.552, P<0.01)$. On day 7, There was a significant positive correlation between IMH size and MI size $(r=0.711$, $P<0.01)$, while no correlation was found between IMH size and ME size $(r=0.429, P=0.097)$.

Conclusions: Infarction size prior to reperfusion is a critical factor in determining whether a Ml is hemorrhagic or non-hemorrhagic in rats.

\section{Background}

Although emergency percutaneous coronary intervention in acute myocardial infarction (MI) restores epicardial coronary blood flow, intramyocardial hemorrhage (IMH) is still observed in up to $50 \%$ of patients [1].Cardiovascular magnetic resonance imaging (CMR) T2* sequence allows accurate visualization of the IMH region than T2 sequence [2-5].

Although IMH and microvascular obstruction (MVO) showed close anatomic correlation [6]. Recently, researchers have shown IMH was more closely associated with adverse outcomes than MVO [7], which is an irreversible pathological consequence of severe reperfused myocardial injury [8]. In the previous studies, hemorrhage occurred only with the largest infarctions [2,7, 9-20], Three studies confirmed a moderate correlation between the extent of necrosis and hemorrhage $[2,19,20]$, but the extent of infarction size of these studies was limited. We hypothesized that IMH could occur in small infarction size and there was a close correlation between IMH size and MI size in small and larger degrees of myocardial ischemia, which can be assessed by the use of CMR images.

In this study, we intended to induce different sizes of myocardial ischemia created by occluding different sections of the proximal left anterior descending coronary artery in a rat model. CMR imaging, including T2, T2*, and LGE was performed on these rats on days 2 and 7 after reperfused myocardial ischemia. The study had four main aims: (1) to find whether IMH presents in small infarction rats; (2) to determine the relationship between of $\mathrm{IMH}, \mathrm{MI}$, and $\mathrm{ME}$ sizes; (3) to compare T2 and T2* values for IMH, MI, ME, and remote area; and (4) to measure changes of $\mathrm{T} 2$ and $\mathrm{T} 2^{*}$ values and sizes of $\mathrm{IMH}, \mathrm{MI}$, and $\mathrm{ME}$ from day 2 to day 7 .

\section{Methods}

\section{Animal models}

This study was approved by the Institutional Animal Care and Use Committee of *BLINDED*. Forty five female Sprague-Dawley rats with body weights between 250 and $350 \mathrm{~g}$ were investigated. To introduce the coronary occlusion, a thoracotomy was performed. The chest was opened at the fourth intercostal space to expose the heart. The pericardium was opened with forceps, and a 6.0 suture was passed underneath the left anterior descending coronary artery (LAD) at 1-3 mm [21-22]. This resulted in occluding the different section of proximal left anterior descending coronary artery leading to different degrees of myocardial infarction $(1,2$, and $3 \mathrm{~mm})$ for each rat. Coronary occlusion was achieved by tightening the suture over a 3.0 suture. All occlusions were maintained for 60 minutes and reperfusion was achieved by untying the knot and releasing the suture from the occlusion [23-24]. Reperfusion was confirmed by ECG changes (ECG, SA Instruments, Inc.).

\section{MRI protocols}


All MRI protocols were implemented on a 7.0T MR system (BRUKER BIOSPEC 70/30). More than five (depending on heart size) singleslice MSME (multi-slice multi-echo)-T2-mapping and MGE (multi-gradient-echo)-T2*-mapping images with the same slices were acquired on the short-axis slices during the mid-diastolic phase and end-inspiratory period using both ECG and respiratory gating systems (SA Instruments, Inc.). Late gadolinium enhancement (LGE) imaging was performed by FISP-cine on the same slice locations at $10 \mathrm{~min}$ after an injection of Gd-DTPA (Magnevist, Bayer Health Care Pharmaceuticals, $0.15 \mathrm{mmol} / \mathrm{kg}$ ).

The imaging parameters included: T2 mapping: TR/TE $=1500 \mathrm{~ms} / 10,20,30 \mathrm{~ms}$, Matrix size $=192 \times 192, \mathrm{FOV}=50 \times 50 \mathrm{~mm}$, and slice thickness $=1.5 \mathrm{~mm}$ without slice gap. T2*-mapping: FA (Flip angle) $=30^{\circ}$, TR/TE=1000ms/3.5,7,10.5,14,17.5,21,24.5,28 ms, Matrix size $=192 \times 192, F O V=50 \times 50 \mathrm{~mm}$, and slice thickness $=1.5 \mathrm{~mm}$ without slice gap. LGE: TR $/ \mathrm{TE}=5.2 \mathrm{~ms} / 1.8 \mathrm{~ms}, F A=25^{\circ}$, matrix size $=256 \times 256$, FOV $=50 \times 50 \mathrm{~mm}$, slice thickness $=1.5 \mathrm{~mm}, 25$ frames for each slice.

\section{Histological}

After the MRI scans, rats were sacrificed with potassium chloride and the hearts were rapidly excised. Each heart was cut into five or more transverse slices from apex to base. Each slice was approximately $1.5 \mathrm{~mm}$ thick in order to match MRI slices. These slices were then incubated in $4 \%$ paraformaldehyde for hematoxylin and eosin staining.

\section{Data Analysis}

According to the $2^{\star}$ results, rats with both $\mathrm{MI}$ and IMH were assigned to a group referred to as the +IMH group, while rats with $\mathrm{MI}$, but no $\mathrm{IMH}$ were assigned to a group that we refer to as the $-\mathrm{IMH}$ group.

T2*- and T2-maps were calculated using a custom-made software written in Matlab 7.1 (The Mathworks). ME was defined as high T2 values (> mean \pm 2 SD in remote normal tissue areas) in T2-maps, Ml areas were identified as positive enhanced areas (> mean $\pm 5 S D$ in remote normal tissue areas) in LGE images. The areas of hemorrhage were identified as a hypointense core (at least 2 standard deviations less than the peri-hemorrhagic myocardium) within a hyperintense territory (confirmed by an LGE-positive area) on T2*-maps. $\mathrm{ME}, \mathrm{MI}$, and IMH sizes were added slice-by-slice, respectively, and expressed as a percentage of the whole myocardial tissue of the left ventricle (\%LV) [25-26].

More than one slice was selected to calculate the mean of T2 and T2* values for each rat. The absolute differences in T2* and T2 were computed as the respective differences in the values between myocardial injury regions-of-interest ( $\mathrm{ME}, \mathrm{MI}$, and IMH) and remote myocardium; they were labeled as T2* and T2. The corresponding relative differences were computed by normalizing the absolute differences using the values from remote regions and multiplying by $100 \%$; they were then labeled as Relative【T2* (\%) and Relative T2 (\%).

All statistical analyses were performed using SPSS Statistics, version 23.0 (IBM Corp.). Interobserver agreement was calculated using the intraclass correlation coefficient. Quantitative data were tested for normal distribution using the Kolmogorov-Smirnov test. Data were expressed as the mean \pm SD or as median ( $25 \%-75 \%$ interquartile range). P-values $<0.05$ were considered significant. The correlations between IMH and the size of MI or ME were assessed using Spearman analysis in the +IMH group and Pearson analysis in the $-\mathrm{IMH}$ group. Datasets following normal distributions (T2 and T2* values) were compared using the analysis of variance with Student-Newman-Keuls post-hoc analysis. When comparing the T2 and T2* values for days 2 and 7, paired-samples t-tests were performed. Comparison between values of size were performed using non-parametric tests.

\section{Results}

\section{Animals}

Thirty five animals were available for imaging (three died during reperfusion, two died during MR scanning, four had no MI, and one had uninterpretable image quality). There were significant differences of $\mathrm{ME}, \mathrm{MI}$, and IMH upon ligating different sections of the proximal left anterior descending coronary artery (table 1 ).

On T2* CMR, hemorrhage was evident in 25 rats (+IMH group) on day 2 and 16 rats (+IMH group) on day 7 (9 rats on day 2 were sacrificed for histological analysis). On the basis of LGE, a total of 167 imaging slices were identified as positive for MI (101 on day 2 and 66 on day 7) and 112 for IMH (66 on day 2 and 46 on day 7). No hemorrhage was observed in ten rats (-IMH group) and all rats on day 2 were sacrificed.. 
Representative LGE images and corresponding noncontrast-enhanced T2*-and T2-maps from animals with and without hemorrhage on days 2 and 7 of $\mathrm{Ml}$ are shown in Figure 1, respectively.

Mean T2* and T2 values of $\mathrm{ME}, \mathrm{MI}, \mathrm{IMH}$, and remote regions, respective relative differences with respect to the remote myocardium (DT2 $[\%]$ and DT2* [\%]), and absolute differences for T2 and T2* (DT2, DT2*), along with comparisons, are given in Table 2.

\section{Relationship in sizes of $\mathrm{IMH}, \mathrm{Ml}$, and ME}

On the $2^{\text {nd }}$ day, $\mathrm{Ml}$ size ranged from $9.5 \%$ to $46.9 \%$, IMH size ranged from $1.0 \%$ to $19.2 \%$, and myocardial edema size ranged from $22.5 \%$ to $55.5 \%$. There was a significant positive correlation between IMH size and MI size or ME size. MI size and ME size of the +IMH group were significantly larger than those of the $-\mathrm{IMH}$ group (table 3 ).

On the 7 th day, $\mathrm{Ml}$ size ranged from $7.9 \%$ to $26.3 \%$, IMH size ranged from $1.6 \%$ to $19.3 \%$, and $\mathrm{ME}$ size ranged from $19.2 \%$ to $38 \%$. There was a significant positive correlation between IMH size and MI size, however, there was no significant correlation between IMH size and ME size. Note that from the 2nd to the 7th day, MI size and ME size decreased, while IMH size remained the same.

\section{T2 and T2* value of IMH, MI,ME}

At day 2, the T2 value of IMH was smaller than that of $\mathrm{MI}$, and bigger than that of the remote area, however, there was no difference between IMH and ME, while on the 7th day, the T2 value of IMH was smaller than that of ME. From the 2nd to the 7th day, the T2 value of $\mathrm{IMH}$ and $\mathrm{MI}$ decreased, but there were no differences between the ME and remote region.

The T2* value of IMH was smaller than that for $\mathrm{Ml}$, and bigger than the remote region; no difference was found between IMH and $\mathrm{ME}$ on the 2nd and 7th day. From the 2nd to 7th day, the T2* value of MI decreased, while there were no changes of ME, IMH, and remote region. The other parameters of T2 and T2* value and the changes from the 2 nd to the 7 th day are shown in table 2 and table 4.

\section{Histopathology}

Representative histopathologic images from animals with hemorrhagic and nonhemorrhagic infarctions sacrificed on the 2nd and 7th day of Ml are shown in Figure 1. In the +IMH group, H\&E stains easily showed evidence of myocardial injury (myocyte necrosis), the distribution of inflammatory cells and hemorrhage. The -IMH group did not show evidence of hemorrhage on H\&E stains.

\section{Discussion}

This is the one of a few studies using CMR to detect the relationship between the extent of MI and hemorrhage in small animal models. We observed that: 1) a small IMH size in the small infarction area, 2) a positive linear relationship between the size of infarction and hemorrhage, 3) the $\mathrm{Ml}$ area was the highest T2 value region, which decreased from $2 \mathrm{~d}$ to $7 \mathrm{~d}$, and 4) the T2 value of IMH decreased from day 2 to 7 , while the size and T2* remained the same.

The infarction size was determined by serval reasons, one was the position of occulusion of coronary artery. One minor discrepancy in the exact location along the LAD where the occlusion was introduced could be lead to a variation of myocardial infarction size [22]. In our study, we used three positions (1-3 mm) of ligating to mimic clinical Ml in patients, while other studies always used about $2 \mathrm{~mm}$ from its origin. We found that in the lowest position of ligating, while the infarction size was the smallest, IMH only occurred in two rats with $2 \%$ and $4.5 \%$ of the whole LV in this group, while $100 \%(11 / 11)$ and $85.7 \%(12 / 14)$ IMH occurred in the highest and middle position ligating groups, respectively.

A patient study revealed a relationship between IMH and $\mathrm{MI}(\mathrm{r}=0.45$ or 0.67$)$, however, the size of $\mathrm{MI}$ was $28.5 \% \mathrm{LV}$ in this study [19]. A recent patient study also reported a relationship between $\mathrm{IMH}$ and necrosis $(\mathrm{r}=0.58)$, and the size of $\mathrm{Ml}$ of this study was $24 \pm 10 \%$ [20] . In a dog study, there was a moderate correlation between the extent of necrosis and the extent of hemorrhage $(r=0.56 ; p<0.05)[2]$, and the size of MI was $19.3 \pm 4 \% \mathrm{LV}$.

Several patient studies found infarction size with IMH than non-IMH [2,7, 9-20] (table 5). While in animal study, However, Hansen ESS Et al reported infarction size (23\% LV) with IMH not than non-IMH in reperfused AMI in 13 pigs (19.7\%LV) [15], A possible explanation for this might be the small sample size ( $n=6$ in IMH group). 
In our study, we designed different extend MI from $9.5 \%$ to $46.9 \%$. This infarction extent was bigger than in former studies [2,7, 9-20]; the range of these studies was not comprehensive to reveal the relationship between IMH and MI. Some areas in our study were smaller than the other study, this suggested that IMH can be found in small MI sizes. This positive correlation suggest that these components of tissue injury are not independent of each other, and that there could perhaps be a causal relationship. Hemorrhage occurred mainly in infarcts with the largest infarct size, suggesting that the amount and severity of tissue injury may be a predisposing factor for the development of hemorrhage. In this study, the infarction area was mainly transmural (20 in 25 rats) in the +IMH group, which also represents severe $\mathrm{Ml}$.

Myocardial hemorrhage was taken to represent a hypointense infarct core with a T2* value of $<20 \mathrm{~ms}$ [7]; in this study, the T2* value of $\mathrm{IMH}$ was 3.2-4.2 ms more than the remote area, and there was no different with the ME region. This is not in line with the former study, while mean $\mathrm{T} 2^{*}$ of the hemorrhagic area was lower than remote myocardium; the possible explanation for this might be different sequence and reperfusion time.

The persisting high T2 value of $\mathrm{MI}$ reflected inflammation around IMH found in this study confirms previous results and indicates that hemorrhage prolongs [27], if not prevents, recovery after 7 days. Our findings are consistent with a former study showing that the noreflow persists for at least 1 month after reopening of the epicardial coronary artery [28]. The MI size decreased from day 2 to day 7 , except for the real myocardial infarction area; another possible explanation for this is that the infarct zone contains a heterogeneous pathology area in early post-Ml.

\title{
Limitations of the study
}

First, there were no quantitative histological data to confirm IMH size; hematoxylin-eosin staining could detect IMH qualitatively to some extent, but lack of accurate method for the whole IMH area. Second, Although the data acquisition window was controlled at a minimum, cardiac motion due to relatively high heart rate remained problematic in some of the rat imaging sessions, especially the $\mathrm{T} 2^{\star}$ sequence. Third, There was only the early time point to detect IMH in reperfused myocardial ischemia. Further studies should be designed to monitor the chronic change of IMH. Last, This study reported IMH only, not MVO; the reason was that FISP-cine with a relatively long acquisition time in the present study may underestimate the extent of MVO.

\section{Conclusion}

In conclusion, Our data suggest that there was a positive relationship between IMH and $\mathrm{MI}$, and the small infarction size could also lead to small IMH, which can not be easily dissolved in the early phase of reperfusion. Myocardial infarction volume could be as an independent predictor of intramyocardial hemorrhage volume for percutaneous coronary intervention of myocardial ischemic patients.

\section{Abbreviations}

\author{
MI myocardial infarction \\ IMH intramyocardial hemorrhage \\ CMR cardiovascular magnetic resonance \\ MVO microvascular obstruction \\ LAD left anterior descending \\ ECG electrocardiogram \\ MSME multi-slice multi-echo \\ MGE multi-gradient-echo \\ FISP fast imaging with steady-state precession
}

TR time of repetition 
TE time of echo

FOV field of view

FA flip angle

ICC intraclass correlation efficient

\section{Declarations}

\section{Authors' contributions}

RX: participated in data acquisition, data analysis, statistical analysis, literature research and drafted the frst manuscript. RX; JZ; YL; FL; FG: conceived the study, participated in its design and coordination, participated in data and statistical analysis and interpretation and helped to draft and revise the manuscript for important intellectual content. TZ; YuZ; BH; YC; LW; YangZ; JL: participated in data acquisition, data analysis and literature research and helped to draft the frst manuscript. YL; FL; FG revised the manuscript for important intellectual content. JZ participated in the study design and critically revised the manuscript for important intellectual content. YZ participated in data analysis, carried out the histological analysis and revised the manuscript for important intellectual. All authors read and approved the final manuscript.

\section{Acknowledgements}

This study was supported by The National Natural Science Foundation of China $(81601546,81520108014,81771800,81829003)$.

\section{Competing interests}

The authors declare that they have no competing interests.

\section{Availability of data and materials}

The datasets used and/or analysed during the current study are available from the corresponding author on reasonable request.

\section{Consent for publication}

Not applicable.

\section{Ethics approval and consent to participate}

The present study was approved by the Institutional Animal Care and Use Committee of Committee on Ethics of Biomedicine, West China Hospital of Sichuan University, Chengdu, PR China.

\section{References}

1. Calvieri C, Masselli G, Monti R, et al. Intramyocardial hemorrhage: an enigma for cardiac MRI? Biomed Res Int 2015:859073.

2. Kumar A, Green JD, Sykes JM, et al. Detection and quantification of myocardial reperfusion hemorrhage using T2*-weighted CMR. JACC Cardiovasc Imaging 2011;4(12):1274-83.

3. Kali A, Tang RL, Kumar A, et al. Detection of acute reperfusion myocardial hemorrhage with cardiac MR imaging: T2 versus T2. Radiology 2013;269(2):387-95.

4. Cokic I, Kali A, Wang X, et al. Iron deposition following chronic myocardial infarction as a substrate for cardiac electrical anomalies: initial findings in a canine model. PLoS One 2013;8(9):e73193.

5. Pavon AG, Georgiopoulos G, Vincenti G, et al. Head-to-head comparison of multiple cardiovascular magnetic resonance techniques for the detection and quantification of intramyocardial haemorrhage in patients with ST-elevation myocardial infarction. Eur Radiol 2021;31(3):1245-56.

6. Robbers LF, Eerenberg ES, Teunissen PF, et al. Magnetic resonance imaging-defined areas of microvascular obstruction after acute myocardial infarction represent microvascular destruction and haemorrhage. Eur Heart J 2013;34(30):2346-53. 
7. Carrick D, Haig C, Ahmed N, et al. Myocardial Hemorrhage After Acute Reperfused ST-Segment-Elevation Myocardial Infarction: Relation to Microvascular Obstruction and Prognostic Significance. Circ Cardiovasc Imaging 2016;9(1):e004148.

8. Konijnenberg LSF, Damman P, Duncker DJ, et al. Pathophysiology and diagnosis of coronary microvascular dysfunction in STelevation myocardial infarction. Cardiovasc Res 2020;116(4):787-805.

9. Mather AN, Fairbairn TA, Ball SG, et al. Reperfusion haemorrhage as determined by cardiovascular MRI is a predictor of adverse left ventricular remodelling and markers of late arrhythmic risk. Heart 2011;97(6):453-9.

10. O'Regan DP, Ariff B, Neuwirth C, et al. Assessment of severe reperfusion injury with T2* cardiac MRI in patients with acute myocardial infarction. Heart 2010;96(23):1885-91.

11. Kandler D, Lücke $C$, Grothoff $M$, et al. The relation between hypointense core, microvascular obstruction and intramyocardial haemorrhage in acute reperfused myocardial infarction assessed by cardiac magnetic resonance imaging. Eur Radiol 2014;24(12):3277-88.

12. Ding S, Li Z, Ge H, et al. Impact of Early ST-Segment Changes on Cardiac Magnetic Resonance-Verified Intramyocardial Haemorrhage and Microvascular Obstruction in ST-Elevation Myocardial Infarction Patients. Medicine (Baltimore) 2015;94(35):e1438.

13. Durighel G, Tokarczuk PF, Karsa A, et al. Acute myocardial infarction: susceptibility-weighted cardiac MRI for the detection of reperfusion haemorrhage at 1.5 T. Clin Radiol 2016;71(3):e150-6.

14. Carrick D, Haig C, Ahmed N, et al. Temporal Evolution of Myocardial Hemorrhage and Edema in Patients After Acute ST-Segment Elevation Myocardial Infarction: Pathophysiological Insights and Clinical Implications. J Am Heart Assoc 2016;5(2):e002834.

15. Hansen ES, Pedersen SF, Pedersen SB, et al. Cardiovascular MR T2-STIR imaging does not discriminate between intramyocardial haemorrhage and microvascular obstruction during the subacute phase of a reperfused myocardial infarction. Open Heart 2016;3(1):e000346.

16. Amier RP, Tijssen RYG, Teunissen PFA, et al. Predictors of Intramyocardial Hemorrhage After Reperfused ST-Segment Elevation Myocardial Infarction. J Am Heart Assoc 2017;6(8):e005651.

17. Bulluck H, Rosmini S, Abdel-Gadir A, et al. Diagnostic performance of T1and T2 mapping to detect intramyocardial hemorrhage in reperfused ST-segment elevation myocardial infarction (STEMI) patients. J Magn Reson Imaging 2017;46(3):877-86.

18. Robbers LFHJ, Nijveldt R, Beek AM, et al. The influence of microvascular injury on native T1 and T2* relaxation values after acute myocardial infarction: implications for non-contrast-enhanced infarct assessment. Eur Radiol 2018;28(2):824-32.

19. Ma M, Diao KY, Yang ZG, et al. Clinical associations of microvascular obstruction and intramyocardial hemorrhage on cardiovascular magnetic resonance in patients with acute ST segment elevation myocardial infarction (STEMI): An observational cohort study. Medicine (Baltimore) 2018;97(30):e11617.

20. Ferré-Vallverdú M, Sánchez-Lacuesta E, Plaza-López D, et al. Prognostic value and clinical predictors of intramyocardial hemorrhage measured by CMR T2* sequences in STEMI. Int J Cardiovasc Imaging. 2021;37(5):1735-44.

21. Chen X, Cui K, Xiu J, et al. Evaluation and simplified measurement of infarct size by myocardial contrast echocardiography in a rat model of myocardial infarction. Int J Cardiovasc Imaging 2009;25(7):713-6.

22. Nair AR, Johnson EA, Yang HJ, et al. Reperfused hemorrhagic myocardial infarction in rats. PLoS One 2020;15(12):e0243207.

23. Xu Z, Chen W, Zhang R, et al. Human Recombinant Apyrase Therapy Protects Against Myocardial Ischemia/Reperfusion Injury and Preserves Left Ventricular Systolic Function in Rats, as Evaluated by 7T Cardiovascular Magnetic Resonance Imaging. Korean J Radiol 2020;21(6):647-59.

24. Chen W, Zhang B, Xia R, et al. T2 mapping at 7T MRI can quantitatively assess intramyocardial hemorrhage in rats with acute reperfused myocardial infarction in vivo. J Magn Reson Imaging 2016;44(1):194-203.

25. Xia R, Lu X, Zhang B, et al. Early Reperfusion Can Reduce Infarction Size but not Salvaged Myocardial Size in Acute Myocardial Infarction Rats. JACC Cardiovasc Imaging 2015;8(5):616-7.

26. Xia R, Lu X, Zhang B, et al. Assessment of myocardial edema and area at risk in a rat model of myocardial infarction with a faster T2 mapping method. Acta Radiol 2015;56(9):1085-90.

27. Wang G, Yang HJ, Kali A, et al. Influence of Myocardial Hemorrhage on Staging of Reperfused Myocardial Infarctions With T2 Cardiac Magnetic Resonance Imaging: Insights Into the Dependence on Infarction Type With Ex Vivo Validation. JACC Cardiovasc Imaging 2019;12(4):693-703. 
28. From the American Association of Neurological Surgeons (AANS), American Society of Neuroradiology (ASNR), Cardiovascular and Interventional Radiology Society of Europe (CIRSE), et al. Multisociety Consensus Quality Improvement Revised Consensus Statement for Endovascular Therapy of Acute Ischemic Stroke. Int J Stroke 2018;13(6):612-32.

\section{Tables}

Table 1 The size parameters of the different ligating left anterior descending coronary artery groups

\begin{tabular}{|lllll|}
\hline & $1 \mathrm{~mm}(\%)$ & $2 \mathrm{~mm}(\%)$ & $3 \mathrm{~mm}(\%)$ & $\mathrm{P}$ \\
\hline $\mathrm{ME}$ & $40.4 \pm 9.4$ & $27.2 \pm 4.1$ & $17.8 \pm 6.4$ & $<0.01$ \\
\hline $\mathrm{MI}$ & $32.5 \pm 8.9$ & $18.8 \pm 5.6$ & $11.5 \pm 3.7$ & $<0.01$ \\
\hline $\mathrm{MH}$ & $9.9 \pm 5.8$ & $3.5 \pm 2.4$ & $0.6 \pm 1.5$ & $<0.01$ \\
\hline
\end{tabular}

Table $2 \mathrm{~T} 2 *$, T2, values of $\mathrm{IMH}, \mathrm{MI}$ and $\mathrm{ME}$ territories and the associated absolute and percent relative differences of the variables with respect to remote myocardium in $2 \mathrm{~d}$ and $7 \mathrm{~d}$

\begin{tabular}{|c|c|c|c|c|}
\hline $2 d$ & $\mathrm{ME}$ & MI & $\mathrm{IMH}$ & remote \\
\hline $\mathrm{T} 2(\mathrm{~ms})$ & $35.6 \pm 3.3$ & $52.6 \pm 9.2$ & $33.8 \pm 8.3$ & \multirow[t]{3}{*}{$26 \pm 2.9$} \\
\hline DT2(ms) & $9.6 \pm 2.7$ & $26.8 \pm 7.5$ & $8.4 \pm 7.4$ & \\
\hline Relative DT2 (\%) & $37.4 \pm 11.8$ & $102.7 \pm 26.6$ & $31.8 \pm 28.6$ & \\
\hline $\mathrm{T} 2 *(\mathrm{~ms})$ & $17.9 \pm 6.6$ & $26.3 \pm 10.3$ & $16.3 \pm 5.9$ & \multirow[t]{3}{*}{$12.3 \pm 4$} \\
\hline $\mathrm{DT} 2 \star(\mathrm{ms})$ & $5.6 \pm 3.4$ & $14 \pm 7.6$ & $4.2 \pm 3.2$ & \\
\hline Relative DT2*(\%) & $46.4 \pm 22.5$ & $119.5 \pm 74.4$ & $35.8 \pm 27.4$ & \\
\hline \multicolumn{5}{|l|}{$7 d$} \\
\hline $\mathrm{T} 2(\mathrm{~ms})$ & $32.7 \pm 3.1(p>0.05)$ & $41.3 \pm 4.5(p<0.01)$ & $27.1 \pm 6(p<0.05)$ & \multirow[t]{3}{*}{$24 \pm 2.1(p>0.05)$} \\
\hline DT2(ms) & $8.7 \pm 1.8(p>0.05)$ & $17.3 \pm 3.6(p<0.01)$ & $3.1 \pm 5.5(p<0.05)$ & \\
\hline Relative DT2 (\%) & $36.3 \pm 7.7(p>0.05)$ & $72.3 \pm 15.5(p<0.01)$ & $12.7 \pm 23.6(p<0.05)$ & \\
\hline $\mathrm{T} 2 *(\mathrm{~ms})$ & $15.5 \pm 2.8(p>0.05)$ & $21 \pm 4.1(p<0.05)$ & $13.9 \pm 2.7(p>0.05)$ & \multirow[t]{3}{*}{$10.6 \pm 1.8(p>0.05)$} \\
\hline $\mathrm{DT}{ }^{\star}(\mathrm{ms})$ & $4.9 \pm 2.2(p>0.05)$ & $10.4 \pm 3.4(p<0.05)$ & $3.2 \pm 2.9(p>0.05)$ & \\
\hline Relative DT2*(\%) & $47.4 \pm 22.8(p>0.05)$ & $99.4 \pm 33.9(p>0.05)$ & $33.2 \pm 30.5(p>0.05)$ & \\
\hline \multicolumn{5}{|l|}{-IMH group } \\
\hline $\mathrm{T} 2(\mathrm{~ms})$ & $39 \pm 7.3(p>0.05)$ & $49.7 \pm 8.7(p>0.05)$ & - & \multirow[t]{3}{*}{$27.1 \pm 3.6(p>0.05)$} \\
\hline DT2(ms) & $11.9 \pm 5(p>0.05)$ & $22.6 \pm 6.4(p>0.05)$ & - & \\
\hline Relative DT2 (\%) & $43.8 \pm 15.5(p>0.05)$ & $83.5 \pm 21.8(p>0.05)$ & - & \\
\hline $\mathrm{T} 2 \star(\mathrm{ms})$ & $15.7 \pm 3.6(p>0.05)$ & $21.9 \pm 4.8(p>0.05)$ & - & \multirow[t]{3}{*}{$10.8 \pm 1.3(p>0.05)$} \\
\hline DT2*(ms) & $4.9 \pm 2.5(p>0.05)$ & $11.1 \pm 4.1(p>0.05)$ & - & \\
\hline Relative DT2*(\%) & $44.5 \pm 18.3(p>0.05)$ & $102.4 \pm 35(p>0.05)$ & - & \\
\hline
\end{tabular}

Data are reported as mean \pm standard deviation. IMH: region of MI containing hemorrhage; MI: is the area hemorrhagic MI devoid of hemorrhage; and ME: non-infarction area. DT2*, DT2 and Relative DT2*, DT2 are as defined in text.

Note. $7 \mathrm{~d} p$ value denote the changes from $2 \mathrm{~d}$ to $7 \mathrm{~d}$. -IMH group $\mathrm{p}$ value denote the differences between two groups. 
Table 3 The size parameters of the +IMH group and -IMH group

\begin{tabular}{|llllll|}
\hline & $2 d\left(M\left(P_{25}, P_{75}\right)\right)$ & $7 d\left(M\left(P_{25}, P_{75}\right)\right)$ & $P$ & -IMH group & $P(v s+I M H 2 d)$ \\
\hline ME & $31.6(26.2,38.4)$ & $23.7(22,32.1)$ & $<0.01$ & $17.3 \pm 6.1$ & $<0.01$ \\
\hline MI & $22(16.7,30.4)$ & $13.8(10.1,20.9)$ & $<0.01$ & $12 \pm 4.4$ & $<0.01$ \\
\hline IMH & $5.1(2.7,9.1)$ & $4.5(3.1,6.7)$ & 0.155 & - & - \\
\hline
\end{tabular}

Table $4 \mathrm{P}$ values of the differences of $\mathrm{T} 2$ and T2* parameters between IMH and other ROIs of +IMH group

\begin{tabular}{|c|c|c|c|c|c|c|c|c|c|c|c|c|}
\hline & $2 d$ & & & & & & $7 d$ & & & & & \\
\hline ROls & $\mathrm{T} 2(\mathrm{~ms})$ & पT2(ms) & $\begin{array}{l}\text { Relative } \\
\text { प्रT2(\%) }\end{array}$ & $\begin{array}{l}\mathrm{T} 2^{\star} \\
(\mathrm{ms})\end{array}$ & $\begin{array}{l}\text { वT2* } \\
(\mathrm{ms})\end{array}$ & $\begin{array}{l}\text { Relative } \\
\text { aाT2 2* } \\
(\%)\end{array}$ & $\mathrm{T} 2(\mathrm{~ms})$ & पT2(ms) & $\begin{array}{l}\text { Relative } \\
\text { प्रT2(\%) }\end{array}$ & $\begin{array}{l}\text { T2* } \\
\text { (ms) }\end{array}$ & $\begin{array}{l}\text { 口T2* } \\
(\mathrm{ms})\end{array}$ & $\begin{array}{l}\text { Relative } \\
\text { पT2*(\%) }\end{array}$ \\
\hline MI & 0.01 & 0.01 & 0.01 & 0.01 & 0.01 & 0.01 & 0.01 & 0.01 & 0.01 & 0.01 & 0.01 & 0.01 \\
\hline ME & $>0.05$ & $>0.05$ & $>0.05$ & $>0.05$ & $>0.05$ & $>0.05$ & 0.01 & 0.01 & 0.01 & $>0.05$ & $>0.05$ & $>0.05$ \\
\hline remote & 0.01 & - & - & 0.05 & - & - & 0.05 & - & - & 0.01 & - & - \\
\hline
\end{tabular}

Table 5 The previous studies found infarction size of IMH was bigger than non-IMH patients

\begin{tabular}{|lll|}
\hline reporters & Infarction size of IMH group $(\% \mathrm{LV})$ & year \\
\hline O'Regan DP et al & $23.8 \pm 8.2$ & 2010 \\
\hline Kumar A et al & $38.8 \pm 2.8$ & 2011 \\
\hline Mather AN et al & 36.2 & 2011 \\
\hline Kandler D et al & $33 \pm 20 \mathrm{ml}(\%$ LV not available) & 2014 \\
\hline Ding S et al & $32.6 \pm 11.6$ & 2015 \\
\hline Durighel G et al & $25(19-28)$ & 2016 \\
\hline Carrick D et al & 29 & 2016 \\
\hline Carrick D et al & $22-30$ & 2016 \\
\hline Bulluck H et al & $33.4 \pm 11.3$ & 2017 \\
\hline Amier RP, et al & $16.9-45.4$ & 2017 \\
\hline Robbers LFHJ et al & $25 \pm 11$ & 2018 \\
\hline Ma M et al & $36.5 \pm 14.8$ & 2018 \\
\hline Ferré-Vallverdú M et al & $24 \pm 10$ & 2021 \\
\hline
\end{tabular}

\section{Figures}



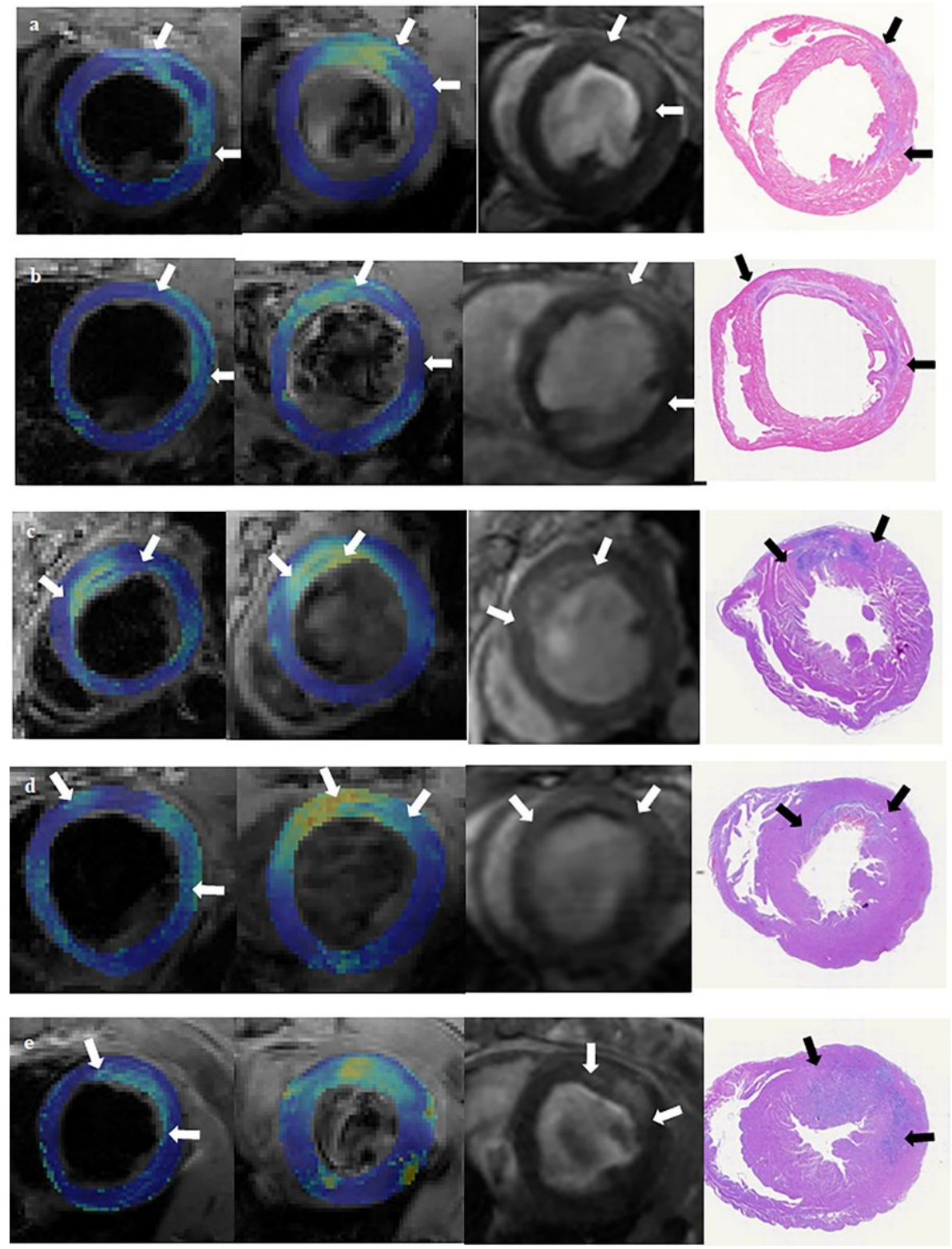

\section{Figure 1}

ME, IMH, MI and myocardial injury areas (arrows) showed by MRI (T2 mapping, T2* mapping, LGE) and H\&E staining separately (from left to right). (a) Bigger IMH and $\mathrm{MI}$ at day 2. (b) Bigger IMH and MI at day 7. (c) Smaller IMH and MI at day 2. (d) Smaller IMH and MI at day 7. (e) non IMH at day 2. IMH, intramyocardial hemorrhage; MI, myocardial infarction; ME, myocardial edema; LGE, late gadolinium enhancement; H\&E, hematoxylin and eosin. 